\title{
The Year-long Flux Variations in Boyajian's Star Are Asymmetric or Aperiodic
}

\author{
Michael Hippke ${ }^{1}$ (10) and Daniel Angerhausen ${ }^{2,3}$ (1) \\ ${ }^{1}$ Sonneberg Observatory, Sternwartestr. 32, D-96515 Sonneberg, Germany; michael@hippke.org \\ ${ }^{2}$ Center for Space and Habitability, University of Bern, Hochschulstrasse 6, 3012 Bern, Switzerland \\ ${ }^{3}$ Blue Marble Space Institute of Science, 1001 4th Avenue, Suite 3201 Seattle, Washington 98154 USA; daniel.angerhausen@csh.unibe.ch \\ Received 2017 October 13; revised 2018 January 25; accepted 2018 January 27; published 2018 February 9
}

\begin{abstract}
We combine and calibrate publicly available data for Boyajian's star, including photometry from ASAS (SN, V, I), Kepler, Gaia, SuperWASP, and citizen scientist observations (AAVSO, HAO, and Burke-Gaffney). Precise (mmag) photometry covers the years 2006-2017. We show that the year-long flux variations with an amplitude of $\approx 4 \%$ cannot be explained with cyclical symmetric or asymmetric models with periods shorter than 10 years. If the dips are transits, their periods must exceed 10 years, or their structure must evolve significantly during each fouryear-long cycle.
\end{abstract}

Key words: stars: variables: general

\section{Introduction}

Boyajian's Star (KIC 8462852) is a mysterious object that showed asymmetric, aperiodic day-long deep (20\%) dips in brightness during Kepler's 2009-2013 mission (Boyajian et al. 2016). The mystery deepened when Schaefer (2016) claimed a dimming of the star during 1890-1990 based on historical plates, and Montet \& Simon (2016) showed that the dimming continued during Kepler's mission. These results have been interpreted as demonstrating that the brightness of Boyajian's Star is monotonically decreasing with time. Although the century-long dimming has been challenged in re-analyses (Hippke et al. 2016; Lund et al. 2016) and with data from multiple other observatories (Hippke et al. 2017), it only recently became clear that the star's brightness shows reoccurring variations with a few percent amplitude on 8-year-long timescales (Simon et al. 2017).

Dimmings and variations are common for young stars (Bodman et al. 2017), but are not known for F3 main-sequence stars (Boyajian et al. 2016). A series of more or less exotic solutions have been proposed, such as the ingestion of a planet (Metzger et al. 2017), intrinsic variations (Foukal 2017), or solar system debris (Katz 2017). To narrow down the model choice, we here examine available data to determine the brightness variations over the last decade.

\section{Method}

\subsection{Observations}

Several independent data sets cover the brightness of Boyajian's star over different intervals. Before the release of the ASAS data, the creation of a consistent light curve was not possible, because few of the other data sets overlap and offsets due to calibration differences were uncertain.

\subsubsection{ASAS-V and ASAS-I}

ASAS is a long-term V- and I-band wide-field variability survey based in Chile (since 1997) and on Hawaii (since 2006; Pojmanski 1997, 2002). For Boyajian's Star, 486 good quality observations were obtained in V- and 247 in I-band between 2006 and 2017 May. Compared to ASAS-SN, ASAS has a longer time baseline, but larger photometric uncertainty
(0.028 mag per observation). This is due to the smaller apertures $(5 \mathrm{~cm}$ versus $14 \mathrm{~cm})$ and lower cadence.

\subsubsection{ASAS-SN}

ASAS-SN is an all-sky V-band transient survey at the same location as ASAS (Shappee et al. 2014). For Boyajian's Star, 377 observations were taken between 2015 February and 2017 May. Aperture photometry was performed by Simon et al. (2017) with a median photometric uncertainty of 0.01 mag per observation.

\subsubsection{SuperWASP}

The WASP was a wide-field white-light survey located on the Canary Islands and South Africa (Pollacco et al. 2006). For Boyajian's Star, a total of 5377 measurements were taken over three observing seasons (2006-2008). The first season with only 22 observations shows 0.2 mag offsets for many stars, and we discard these data. The remaining data cover the time intervals 2007.41-2007.51 and 2008.36-2008.59.

\subsubsection{Kepler FFI}

Kepler photometry is optimized to detect small, short duration signals such as planet transits at the expense of long-term trends. Long-term variability ( $>30$ days) can be recovered in photometry from the full frame images (FFIs), as shown by Montet et al. (2017), Montet \& Simon (2016), and Montet (2017). A total of 52 such measurements could be extracted for Boyajian's Star, with a typical uncertainty of $0.001 \mathrm{mag}$.

\subsubsection{Citizen Science Observations}

Citizen scientists from the American Association of Variable Star Observers (AAVSO) collected $>30,000$ V-band observations between 2015.7 and 2017.8 with varying quality. We tried different quality cuts such as only taking observations with low estimated uncertainties or only observations from observers with a minimum number of images. Compared to the full data set, all tested quality cuts yield very similar results within a few millimag when taking monthly bins. We choose to 


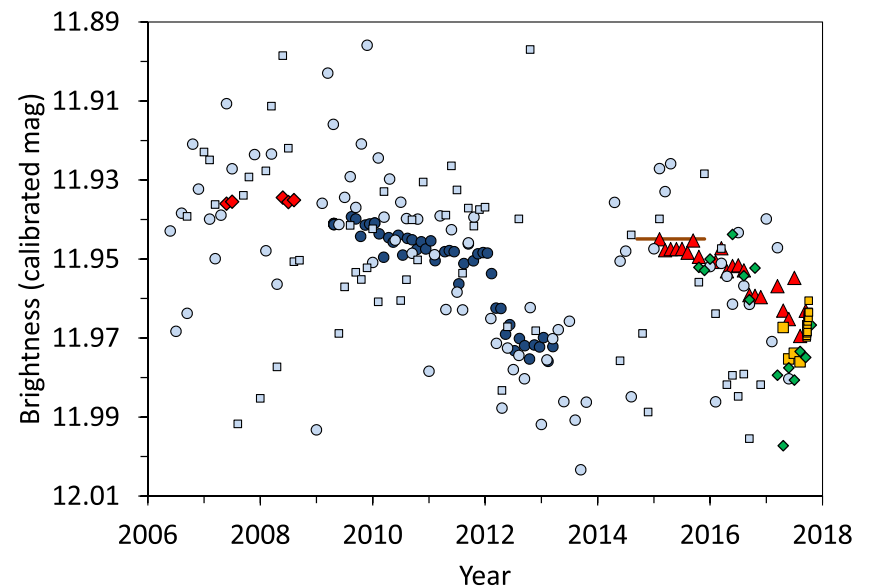

Figure 1. Timeseries overview of all data used in this analysis: ASAS-I (light blue squares), ASAS-SN (red triangles), SuperWASP (red diamonds), Gaia (line), AAVSO and LDJ (green diamonds), and HAO (orange squares)

only eliminate observations that differ by more than $5 \sigma$ from the mean of a month, and show the monthly bins in Figure 1.

Citizen scientist Bruce Gary of Hereford Arizona Observatory publicly released 75 observations ${ }^{4}$ mostly in V-band taken between 2017.3 and 2017.8 with a typical uncertainty of 0.002 mag. Starting in 2017 October, observations were taken in $\mathrm{g}^{\prime}$-band with a date-average uncertainty of $0.001 \mathrm{mag}$.

Citizen scientist Dave Lane (LDJ) of Burke-Gaffney Observatory provided 520 observations taken with a Planewave CDK24 telescope (aperture $0.61 \mathrm{~m}$, focal length $3970 \mathrm{~mm}$ ) located in Halifax, Canada, with an Apogee CG16M CCD camera $(\mathrm{KAF}-16803$ sensor binned $2 \times 2)$ and a $50 \mathrm{~mm}$ square Astrodon V filter. Observations from 2016 May 1 to 2017 September 23 (HJD 2457509.6 to 2458019.6) were differential photometry using the single AAVSO comparison star 000-BLS-549 (mag = 12.427). Subsequent observations are ensemble photometry using five AAVSO comparison stars.

\subsubsection{Gaia}

Variability information is not released in Gaia's DR1 (Gaia Collaboration et al. 2016), but it has been shown by Belokurov et al. (2017) that variability can be approximated from the average measured flux and the reported uncertainty. As shown by Simon et al. (2017), Boyajian's star is less variable than 11 of 14 comparison stars between 2014 July and 2015 September, which have an average scatter of $1.6 \%$. We therefore show the Gaia data as a line in Figure 1, noting that the width of this line is uncertain until Gaia's DR4, which will include the photometry.

\subsection{Normalization}

In order to compare all data sets to each other and eventually combine them to one overall timeseries, a benchmark is needed. Fortunately, the ASAS-V and ASAS-I cover the entire timeline from 2006 to the present and can be used as a reference zero-point value for the other data sets. A comparison of $\mathrm{V}$ to I shows that the I-band has fewer observations, larger uncertainties and is less affected from the brightness variations we seek to analyze here. Therefore, we choose the ASAS-V

\footnotetext{
http://www.brucegary.net/ts4/
}

data as our baseline. The other data sets are calibrated by normalizing their flux values with respect to ASAS-V, so that the squared residuals are minimized for the overlapping segments. We tested the nearest-neighbor method (in time), splines, and monthly bins and got virtually identical results. We proceed by using the calibration to monthly bins as shown in Figure 1 for maximum simplicity. The combined light curve suffers from bandpass differences. We assume, however, that color-differences during the variations have amplitudes that are on much smaller scales than the overall brightness changes. For example Boyajian et al. (2018) and Deeg et al. (2018) showed that even for the strong short-term dimmings, that make Kic 846 so peculiar, the difference between very different bandpasses such as $\mathrm{r}$ and $\mathrm{B}$ is only a few tenths of a percent, while all bandpasses clearly show the dimmings on a several percent level (Figure 7 in their paper). Most of our data consists of $\mathrm{V}$ or white light measurements that we use to analyze longterm trends and cyclical variation. In general, as our results rely on the qualitative fit, slight differences at the millimag level are irrelevant. While calculations were made on the individual data points, we show monthly bins in the figures for better visibility. Despite the larger scatter in ASAS-V and ASAS-I compared to the other data sets, their baseline is helpful because they span the entire time from 2006 to 2017.

Using ASAS data, Simon et al. (2017) found indications for cyclical brightness variations with a period of $\approx 8$ years. The variability is color-dependent. The dimming is less in UV $(0.2 \mu \mathrm{m})$ than IR $(4.5 \mu \mathrm{m})$, so that the responsible bodies must be small (microns) in size (Meng et al. 2017). The brightening seen in ASAS-V is not clearly visible in ASAS-I, also noted by Simon et al. (2017). These bandpass variations are also evident in our combined light curve, so that the true amplitudes may be incorrect by up to $20 \%$, depending on color.

There is also a significant overlap between ASAS-SN, AAVSO, and HAO; and the gap between SuperWASP and Kepler is short (8 months). As can be seen in Figure 1, all data sets can consistently be coadded into a combined light curve. The systematic calibration uncertainty is small: the SuperWASP average is 11.935 mag when calibrated to ASAS-V and would be $11.941 \mathrm{mag}$ when taking the closest Kepler point as reference, a difference of $0.6 \%$ in brightness. A similar uncertainty exists for the ASAS-SN, AAVSO, and HAO data sets at the level of $0.1 \%-0.3 \%$. These variations are mostly due to the bandpass differences and do not affect our qualitative results.

\section{Results and Model Discussion}

In Figure 1, we show the 2006-2017 long-term photometry of KIC 8462852. Using the ASAS-V monitoring data as a baseline, we were able to combine all other photometric data available to us at the time of submission. The plot shows various levels of variation, which we discuss deeper in the following subsections.

\subsection{Shortest Possible Period Symmetric Model}

Using the highest-quality, uninterrupted FFI data from Kepler (covering 2009-2013), we create the shortest possible period symmetrical flux model (Figure 2). The curve has been created by fitting two polynomials to the "pre-ingress" and the "ingress" time. With the shape of the curve in this model, we only aim to determine the duration and amplitude of the signal. 


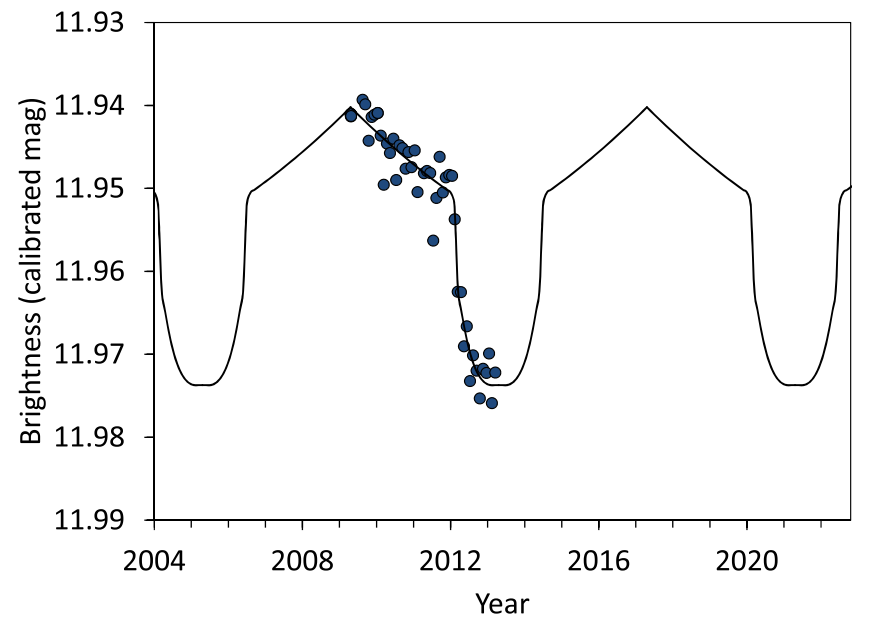

Figure 2. Shortest possible period symmetrical model (line) using Kepler data (blue circles).

This curve covers 8 years in time, compatible to the $\approx 8$-yearlong cycle found by Simon et al. (2017).

For the shortest possible brightness recovery, we stitch a mirror-image of the ingress section immediately to the end of the Kepler data. The shape of this curve is purely phenomenological, i.e., it represents the data well, but does not attempt to explain any underlying physical cause. Qualitatively, it resembles a transit-like signal comparable to year-long eclipsing binaries (Rodriguez et al. 2016), which could be tested spectroscopically.

The issue with this model is that all data sets other than Kepler do not fit into it (Figure 3, left). The steady flux from SuperWASP during 2007-2008 is in disagreement with the expected brightening. Also, the $\approx 2 \%$ dimming seen by ASASSN, AAVSO, and HAO between 2015 and 2017 is against the expected slight brightening for this time. Clearly, the tested period of 8 years is too long, approximately by a factor of two. This cannot be rectified by making the period longer or increasing the transit duration.

\subsection{Shortest Possible Period Asymmetric Model}

If the model is asymmetric, it can be shorter. We keep the well-defined first half of the model, but replace the "egress" with a steep and short curve (Figure 3, right). Now, the second dimming (2015-2017) is well represented in the model. The issue is, however, in the SuperWASP data for the years 2007-2008, which exclude the expected presence of a dip. Instead, there is a hint of a dip at the very beginning of ASAS-V data (Figure 1) in early 2006, about one year earlier than predicted by the asymmetric model. This could be interpreted as an aperiodic cycle.

We may also challenge the validity of the SuperWASP data, which have been discussed in Hippke et al. (2017, their Figure 7). In brief, these data contain 5355 individual measurements covering 2007.41-2007.51 and 2008.36-2008.59. The brightness is constant at the millimag level during this time. The question of the relative brightness compared to the other data sets remains, as each bandpass is different. SuperWASP ended only 8 months before Kepler started, so that it appears plausible, although uncertain, that they share a similar baseline. If this connection is dropped, we are left with the average flux of ASAS for the respective time. Between 2007.41 and 2008.59, there are 27 individual measurements from ASAS-V with an average, constant brightness of $11.935 \pm 0.005 \mathrm{mag}$. The result is identical if all 45 measurements for the years 2007 and 2008 are combined. This represents a brightness uncertainty of $0.5 \%$ from ASAS-V alone. The dip in the asymmetric model would require a dimming down to at least 11.97 in this bandpass, which is in conflict with the ASAS-V observations by $7 \sigma$. When we repeat a similar analysis for the ASAS-I data for 2007-2008, we obtain an average brightness of $11.94 \pm 0.007 \mathrm{mag}$, which is consistent with ASAS-V and in conflict with the dip by $4 \sigma$.

Therefore, we argue that the asymmetric model cannot reproduce the data, because a dip would be expected during 2008, which was not observed. We conclude that neither a symmetric nor an asymmetric model can be periodic on timescales shorter than 10 years (2007-2017). Of course, longer periodic models are still possible.

\subsection{Similarity of the Dimming Slopes 2012 and 2017}

Despite the impossibility of the 4-year symmetric and asymmetric models, the repetition of the dimming slope is remarkable. We achieve the best match to the Kepler FFI data (blue symbols in Figure 4) with a shift of 4.7 years (1700 days), although shifts as short as 1600 days are visually similar. The latest HAO, AAVSO, and ASAS-SN data indicate a steep rebrightening starting in early 2017 October. If this trend continues, the star could return back to maximum brightness by the end of 2017 (dashed line in Figure 4). If the cycle is instead asymmetric, the flux would not follow this line; such a hypothesis can be put to the test in late 2017/early 2018 .

Sacco et al. (2017) recently compared the day-long dips from 2013 and 2017 and found similar structures with a period of 1574 days (4.31 years). This is broadly compatible with our time lag. Unfortunately, there is not much data available between late 2016 and early 2017 (because the star was difficult to observe due to it being close to the Sun) to track down the exact beginning of the steep decline.

Simon et al. (2017) suggest the presence of an 8-year sinusoidal trend based on the ASAS-V and ASAS-I observations (compare light blue symbols in Figure 1). We can reproduce this cycle with an FFT and a periodogram of the data, but the issue here is that the data covers only 10.93 years, or little more than one such cycle. If an underlying strictly periodic phenomenon exists, and it contains asymmetric structure within, it might be longer and non-sinusoidal. For a robust periodicity analysis, data for several cycles (at least 2-3) are needed.

\section{Conclusions}

Wright \& Sigurdsson (2016) described a number of potential explanations for this object's behavior, such as clouds in the outer solar system, structure in the interstellar medium (ISM) along the line of sight, natural and artificial material orbiting Boyajian's Star, an intervening object with a large disk or ring, and stellar variations. They conclude that the ISM and intervening disk models are the more plausible ones. Several other groups picked up from there and analyzed individual phenomena in more detail and in the light of new observations. Here we discuss these in the context of our findings.

Foukal (2017) discussed internal stellar effects that potentially explain the flux obstruction. They suggest that magnetic activity, differential rotation, changes in photospheric 

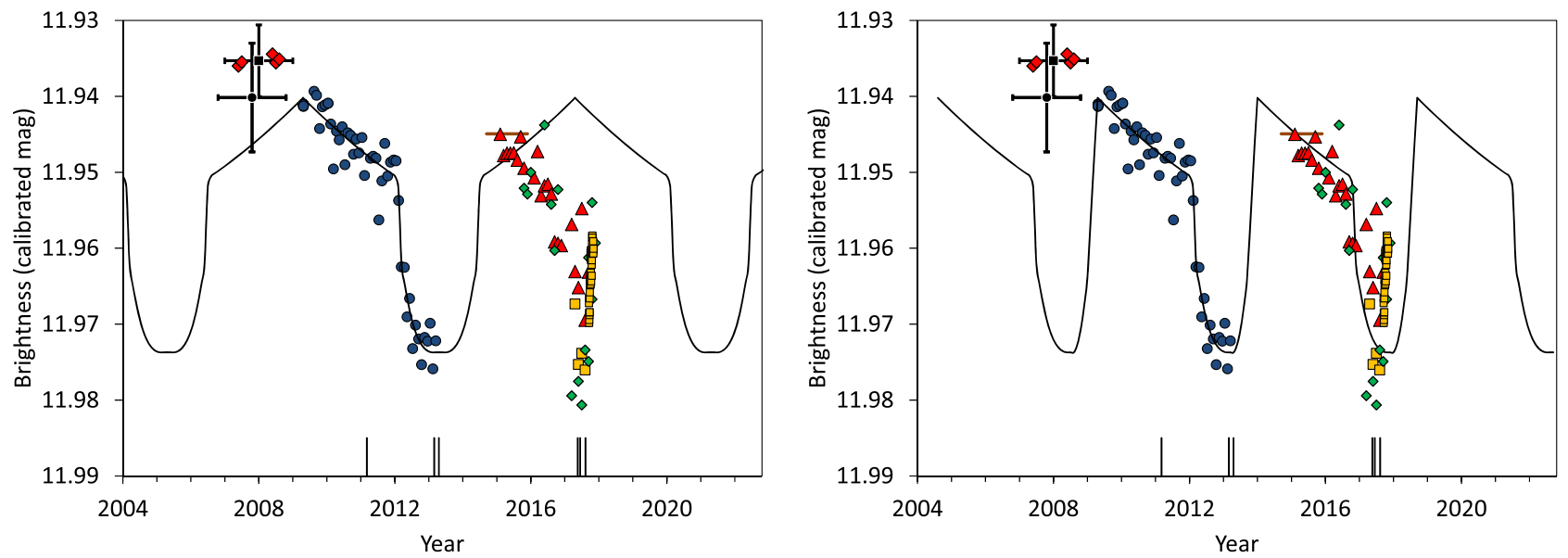

Figure 3. Left: the symmetric model is incompatible with calibrated data. Right: the asymmetric model is incompatible with SuperWASP data (red triangles), which were calibrated to ASAS-V (upper points with uncertainties) and ASAS-I (lower points with uncertainties). The deepest day-long dips are marked with vertical lines.

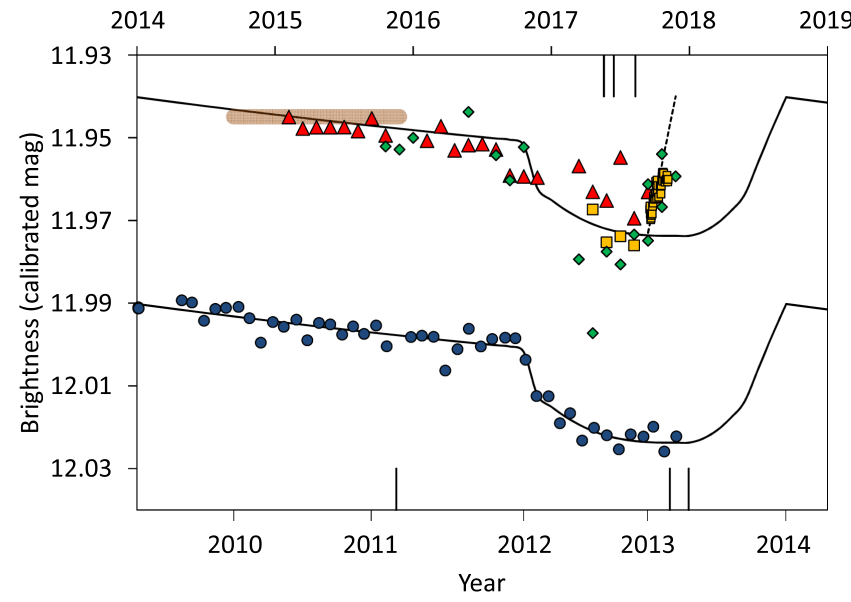

Figure 4. Comparison of Kepler FFI dimming (lower half and ordinate) and recent data (upper part). The cycle shown here is 4.7 years and appears remarkably similar. It is currently (2017 October) unclear if the star is already rebrightening (dashed line).

abundances, or just random variation in convective efficiency could produce effects such as the ones observed. Such effects, or a combination of them, could be periodic, aperiodic, symmetric, or asymmetric. They can, however, be tested by multicolor photometry, for which observations are ongoing.

As can be seen in Figures 3 and 4, the times of the deepest dips occur just before the subsequent brightening. This might provide interesting evidence that the brightening is caused by internal storage of the blocked energy in the short dips.

Katz (2017) posed the question of whether the structure in KIC 8462852 could have been caused by matter in the solar system, considering heliocentric obscuring rings in the outer solar system that graze the line of sight to the star once per orbit of Kepler. While such a phenomenon is not impossible, it is presently unknown to appear in other stars, and should be periodic at first order. We see the photometry presented in this paper as evidence against such solar system rings.

Ballesteros et al. (2017) proposed that a giant planet orbits KIC 8462852, which hosts a set of rings as well as two massive clouds of trojan asteroids in the planet's Lagrange points on its orbit. Such a complex system could well cause multiple asymmetric dips, such as those observed here, depending on its inclination, eccentricity, and overall geometry. Trojans near
L4 and L5 can have, in addition, different shapes and sizes, which would be in orbit around their Lagrange points, and thus produce very different transits, or sometimes none at all. Such a system can be constrained with our analysis to a period of at least a decade, and could be probed with transit spectroscopy.

Metzger et al. (2017) theorized a post-merger return to normal after the ingestion of a planet up to 10,000 years ago. Gravitational energy released as the body spiraled into the outer layers of the star could have caused a temporary and unobserved brightening, which would explain the (disputed) 100 -year dimming. The individual transient dimming events would then be caused by planetary debris from an earlier partial disruption of the same bodies, or due to evaporation and outgassing from a tidally detached moon system. Alternatively, they discuss, similar to the detection paper, that the dimming events could arise from a large number of comet- or planetesimal-mass bodies placed on high-eccentricity orbits by the same mechanism. This model can only weakly be constrained with our analysis, but its effects should fade over time.

Regarding the small-scale ISM structure, Makarov \& Goldin (2016) discuss a foreground swarm of comet-like objects or planets crossing the line of sight to the star and its optical companions at approximately 7 mas per year as a more plausible interpretation than a family of highly eccentric comets orbiting the target star. The swarm may be a freetraveling interstellar group of objects or a belt associated with an additional hypothetical optical source. Again, our data can only weakly constraint this model, but its effects should fade over time. Since the review of Wright \& Sigurdsson (2016), the study by Meng et al. (2017) favored circumstellar over ISM obscuration based on new multicolor photometry.

\section{Conclusion}

We have combined and calibrated publicly available data for Boyajian's star. All data are consistent with each other and shows prominent dimming events with an amplitude of $\approx 4 \%$ in 2012 and 2017, which are remarkably similar in time and depth. Due to the short time in between these events, a symmetric light curve cannot be constructed. An asymmetric periodic model is limited by the fact that no dimming was seen during 2007 in ASAS-V $(7 \sigma)$, ASAS-I $(4 \sigma)$, and SuperWASP at high confidence. If the dimmings are periodic, their period 
must exceed 10 years. Clearly more observational data are needed to solve this puzzle and we encourage the professional as well as citizen science community to continue their great effort in photometric monitoring.

We are thankful to Bruce Gary, Dave Lane, the AAVSO observers, and the ASAS team for obtaining and providing data. We thank the referees for their comments that improved the paper.

\section{ORCID iDs}

Michael Hippke (1) https://orcid.org/0000-0002-0794-6339

Daniel Angerhausen (iD https://orcid.org/0000-0001-

6138-8633

\section{References}

Ballesteros, F. J., Arnalte-Mur, P., Fernandez-Soto, A., \& Martinez, V. J. 2017, MNRAS, 473, L21

Belokurov, V., Erkal, D., Deason, A. J., et al. 2017, MNRAS, 466, 4711

Bodman, E. H. L., Quillen, A. C., Ansdell, M., et al. 2017, MNRAS, 470, 202

Boyajian, T. S., Alonso, R., Ammerman, A., et al. 2018, ApJL, 853, L8

Boyajian, T. S., LaCourse, D. M., Rappaport, S. A., et al. 2016, MNRAS, 457,3988
Deeg, H. J., Alonso, R., Nespral, D., \& Boyajian, T. 2018, A\&A, in press (arXiv:1801.00720)

Foukal, P. 2017, ApJL, 842, L3

Gaia Collaboration, Brown, A. G. A., Vallenari, A., et al. 2016, A\&A, 595, A2

Hippke, M., Angerhausen, D., Lund, M. B., Pepper, J., \& Stassun, K. G. 2016, ApJ, 825, 73

Hippke, M., Kroll, P., Matthai, F., et al. 2017, ApJ, 837, 85

Katz, J. I. 2017, MNRAS, 471, 3680

Lund, M. B., Pepper, J., Stassun, K. G., Hippke, M., \& Angerhausen, D. 2016, ApJ, submitted (arXiv:1605.02760)

Makarov, V. V., \& Goldin, A. 2016, ApJ, 833, 78

Meng, H. Y. A., Rieke, G., Dubois, F., et al. 2017, ApJ, 847, 131

Metzger, B. D., Shen, K. J., \& Stone, N. 2017, MNRAS, 468, 4399

Montet, B. T. 2017, f3: Full Frame Fotometry for Kepler Full Frame Images, Astrophysics Source Code Library, ascl:1705.006

Montet, B. T., \& Simon, J. D. 2016, ApJL, 830, L39

Montet, B. T., Tovar, G., \& Foreman-Mackey, D. 2017, ApJ, 851, 116

Pojmanski, G. 1997, AcA, 47, 467

Pojmanski, G. 2002, AcA, 52, 397

Pollacco, D. L., Skillen, I., Collier Cameron, A., et al. 2006, PASP, 118, 1407

Rodriguez, J. E., Stassun, K. G., Lund, M. B., et al. 2016, AJ, 151, 123

Sacco, G., Ngo, L., \& Modolo, J. 2017, arXiv:1710.01081

Schaefer, B. E. 2016, ApJL, 822, L34

Shappee, B. J., Prieto, J. L., Grupe, D., et al. 2014, ApJ, 788, 48

Simon, J. D., Shappee, B. J., Pojmanski, G., et al. 2017, ApJ, in press (arXiv:1708.07822)

Wright, J. T., \& Sigurdsson, S. 2016, ApJL, 829, L3 